\title{
Selection of Carpenter Manufacturer using Fuzzy EDAS Method
}

\author{
Zeljko Stevic ${ }^{1}$, Marko Vasiljevic ${ }^{1}$, Edmundas Kazimieras Zavadskas ${ }^{2}$, Sinisa Sremac ${ }^{3}$, Zenonas \\ Turskis $^{2}$
}

${ }^{1}$ University of East Sarajevo

Vojvode Misica 52, 74 000, Doboj, Bosnia and Herzegovina

E-mail.zeljkostevic88@yahoo.com,drmarkovasiljevic@gmail.com

${ }^{2}$ Vilnius Gediminas Technical University

Sauletekio al. 11, LT-10223 Vilnius, Lithuania

E-mail.edmundas.zavadskas@vgtu.lt,zenonas.turskis@vgtu.lt

${ }^{3}$ University of Novi Sad

Trg Dositeja Obradovica 6, 21000 Novi Sad, Serbia

E-mail.sremacs@uns.ac.rs

cross $^{\text {ref }}$ http://dx.doi.org/10.5755/j01.ee.29.3.16818

\begin{abstract}
Making a decision in everyday life always comes with uncertainty and responsibility. To reduce the risk to a minimum and to make the right decision, a person can use methods of multi-criteria analysis in combination with fuzzy logic. A married couple, representing decision-makers in this case study, have purchased an apartment and it needs to be completely refurbished including outside carpentry. The aim of this study is to select the most suitable manufacturer of PVC carpentry for the apartment refurbishing. A total pool of 14 quantitative and qualitative criteria is used as a base for the selection of the most suitable manufacturer of the seven available. For this case study, we will use one of the newer methods - - multicriteria analysis of fuzzy Evaluation Based on Distance from Average Solution (fuzzy EDAS) method. After obtaining the results, an analysis of sensitivity has been conducted showing the stability of results where manufacturer number 4 represents an optimal solution in 13 experimental sets out of 14 in total.
\end{abstract}

Keywords: Multi-Criteria Decision Making; Fuzzy Evaluation Based on Distance from Average Solution (Fuzzy EDAS); Manufacturer; Decision-Maker; Trapezoidal Fuzzy Number; Research.

\section{Introduction}

Psychology provides an explanation as to why individuals frequently make irrational decisions, while economics provide normative theories (Morselli, 2015). According to Chen et al., (2015) multi-criteria decisionmaking (MCDM) is an effective systematic and quantitative way to deal with vital real-life problems in the presence of a number of alternatives and several (conflicting) criteria. A great number of works applying diverse MCDM techniques for engineering problems have been published recently (Zavadskas et al., 2016). Everyday use of MCDM methods (Mardani et al., 2015; Gul et al., 2016, Bojanic et al. 2018) has certainly contributed to the rise in popularity of this area (Zavadskas et al., 2014). Many of hybrid MCDM models are proposed to solve different problems in engineering. An integrated fuzzy AHP and fuzzy MOORA approach is applied to solve the problem of industrial engineering sector (Akkaya et al., 2015). One of the areas where these methods are often applied are location problems where it is possible to rank and select the location of various applications, such as location selection of construction site (Jelokhani-Niaraki \& Malczewski, 2015; Turskis et al., 2015), or industrial site selection (Rikalovic et al., 2014). The following multicriteria problems are also solved, namely, in relation to transport (Stevic et al. 2017a; Pamucar \& Cirovic, 2015; Macharis \& Bernardini, 2015), and in relation to supplier selection (Govindan et al., 2015; Stevic et al., 2016a,
Vasiljevic et al. 2018). Some other areas where the application of these methods is less present but not less important are: ranking municipal solid waste treatment alternatives (Soltan et al., 2015), construction solutions for energy efficient single-family house (Motuziene et al., 2016), the selection of construction projects (Ulubeyli \& Kazaz, 2016), assessment of housing market sustainability (Nuuter et al., 2015), partner selection (Akhavan et al., 2015), choosing residential building repair variants (Bucoń \& Sobotka, 2015), technical and economic assessment of revitalization aspects of down-town tenement-houses (Marcinkowska et al., 2015), evaluation of building foundation alternatives (Turskis et al., 2016), choice of electricity generation technologies (Streimikiene et al., 2016), fire risk assessment (Pushkina et al., 2015), etc. The evaluation of criteria during the selection of proper alternative in construction is not an easy task due to the lack of up-to-date data and indicators measuring problems (Zavadskas et al., 2014; Medineckiene et al., 2015). These problems involve a multitude of requirements and uncertain conditions that have to be taken into consideration simultaneously (Zavadskas et al., 2015).

The main aim of this paper is to make a decision on selecting the best manufacturer of PVC carpentry for the apartment refurbishment taking into account the uncertainties and inaccuracies that emerge in such processes. Thus, in this paper, the application of fuzzy EDAS method 
provides support in solving important issues for everyday functioning. In this multi-criteria model, making a decision on the best manufacturer has a significant influence on the quality of life of the decision-maker since the choice of the proper alternative provides easier and simpler functioning.

The authors decided to apply the fuzzy EDAS method for several reasons. The method allows the calculation of criteria weights, which is not the case with other multicriteria decision-making methods. If any other method is used, e.g. fuzzy CORPAS or fuzzy ARAS, integration with the criteria weighting method such as AHP or BWM would have to be performed. In addition, in this paper, the multicriteria model is based on 14 criteria, and using the fuzzy EDAS method it is possible to determine their significance in a very simple and easy way, while it is not the case with other methods. If the AHP or BW method were to be applied, then a hierarchical structure of criteria with different levels would have to be formed and that would further complicate the proposed model. The third reason for applying the fuzzy EDAS method is its great application so far. According to Stevic et al. (2017b), in a very short time it has found its way through the wide application in solving engineering problems as well as problems in business decision-making. Several studies have already been published in different fields where this method has been applied in its traditional form or some other forms (Keshavarz Ghorabaee et al., 2018; Trinkuniene et al., 2017; Stanujkic et al., 2017; Keshavarz Ghorabaee et al., 2017; Kahraman et al., 2017; Turskis \& Juodagalviene, 2016; Stevic et al., 2016b). Furthermore, one of the reasons for using EDAS method, according to Stevic et al. (2017b), is a mathematical apparatus which assumes the evaluation of alternatives on the basis of positive and negative deviations from the average solution. Such model presents a very important support in decision-making in everyday conflict situations.

Apart from the introduction and conclusion, the paper comes in three chapters. The first chapter shows materials and methods used within the framework where basic setups of fuzzy logic, required for fuzzy EDAS method, have been set. Basic operations with fuzzy trapezoid numbers used in this method and all the necessary steps of the applied method have been shown. Chapter two shows some practical research, where the beginning gives an explanation to the problem being solved, potential alternatives and formed criteria, followed by actual problem solving and display of obtained results. Chapter three is the analysis of sensitivity completed with the use of 14 sets where criteria have different values, and is used as a base of stability of the results. Finally, conclusions with the views on the aim of the work and future research for similar problems end the paper.

\section{Material and Methods}

\section{Fuzzy Sets}

Fuzzy sets are sets whose elements have degrees of membership. The theory of fuzzy sets was first introduced by Zadeh, (1965), whose application enables decision-makers to effectively deal with the uncertainties. In a classical set theory, the membership of elements in a set is assessed in binary terms according to a bivalent condition - an element either belongs or does not belong to the set. Fuzzy sets use generally triangular (TFN), trapezoidal and Gaussian fuzzy numbers, which convert uncertain fuzzy numbers. Some of the definitions related to fuzzy sets and fuzzy numbers, which are used to fuzzy EDAS method, are stated as follows:

Definition 1. A fuzzy subset $\tilde{A}$ of a universal set $X$ can be defined by its membership function $\mu \tilde{\mathrm{A}}(\mathrm{x})$ as: (Zimmermann, 2010);

$$
\tilde{A}=\left.\left(x_{*} \mu_{A}(x)\right)\right|_{x \in X}
$$

where $x \in X$ denotes the elements belonging to the universal set, and $\mu \tilde{\mathrm{A}}(\mathrm{x}): \mathrm{X} \rightarrow[0,1]$.

Definition 2. A fuzzy number is a special case of a convex, normalized fuzzy subset $(\sup \mu \tilde{\mathrm{A}}(\mathrm{x})=1)$ of the real line $\mathrm{R} \mu \tilde{\mathrm{A}}(\mathrm{x}): \mathrm{R} \rightarrow[0,1]$. (Wang and Lee, 2007);

Definition 3. A fuzzy number $\tilde{\mathrm{A}}$ is a trapezoidal fuzzy number (TFN) if its membership function is: (Olcer and Odabasi, 2005);

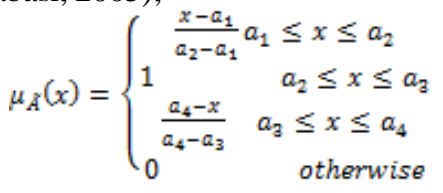

Definition 4. A crisp number $\mathrm{k}$ can be represented by a trapezoidal fuzzy number $\tilde{\mathrm{k}}=(\mathrm{k}, \mathrm{k}, \mathrm{k}, \mathrm{k})$.

Definition 5. Suppose that $A=\left(a_{1}, a_{2}, a_{2}, a_{4}\right)$ and $\hat{B}=\left(b_{1}, b_{2}, b_{2}, b_{4}\right)$ are two positive trapezoidal fuzzy numbers $\left(a_{1} \geq 0\right.$ and $\left.b_{1}>0\right)$ and $k$ is a crisp number. The arithmetic operations with these fuzzy numbers are defined as follows: (Chen and Hwang, 1992);

Addition:

$$
\begin{aligned}
& \widetilde{A} \oplus \bar{B}=\left(a_{1}+b_{1}, a_{2}+b_{2}, a_{2}+b_{2}, a_{4}+b_{4}\right) \\
& \tilde{A}+k=\left(a_{1}+k_{v} a_{2}+k_{v} a_{3}+k_{v} a_{4}+k\right) \\
& \text { Subtraction: } \\
& \widetilde{A} \ominus B=\left(a_{1}-b_{4}, a_{2}-b_{2}, a_{2}-b_{2}, a_{4}-b_{1}\right) \\
& \tilde{A}-k=\left(a_{1}-k, a_{2}-k, a_{a}-k, a_{4}-k\right) \\
& \text { Multiplication: } \\
& \widetilde{A} \otimes B=\left(a_{1} \times b_{1}, a_{2} \times b_{2}, a_{a} \times b_{3}, a_{4} \times b_{4}\right) \\
& \widetilde{A} \times k=\left\{\begin{array}{l}
\left(a_{1} \times k, a_{2} \times k_{s} a_{9} \times k_{s} a_{4} \times k\right) \text { if } k \geq 0 \\
\left(a_{4} \times k, a_{3} \times k_{s} a_{2} \times k_{v} a_{1} \times k\right) \text { if } k<0
\end{array}\right.
\end{aligned}
$$

Division:

$$
\begin{aligned}
& A \bigcirc B=\left(a_{1} / b_{4}, a_{2} / b_{2}, a_{2} / b_{2}, a_{4} / b_{1}\right) \\
& A / k=\left\{\begin{array}{l}
\left(a_{1} / k, a_{2} / k_{v} a_{a} / k_{v} a_{4} / k\right) \text { if } k>0 \\
\left(a_{4} / k_{v}, a_{3} / k_{v} a_{2} / k_{v} a_{1} / k\right) \text { if } k<0
\end{array}\right.
\end{aligned}
$$

Definition 6. Let $A=\left(a_{1}, a_{2}, a_{\mathrm{g}}, a_{4}\right)$ be a trapezoidal fuzzy number. Then, the defuzzified (crisp) value of this fuzzy number can be defined as follows: (Keshavarz et al., 2014)

$$
k(A)=\frac{1}{a}\left(a_{1}+a_{2}+a_{a}+a_{4}-\frac{a_{3} \times a_{4}-a_{1} \times a_{2}}{\left(a_{3}+a_{4}\right)-\left(a_{1}+a_{2}\right)}\right)
$$

Definition 7. Suppose that $A=\left(a_{1}, a_{2}, a_{3}, a_{4}\right)$ is a trapezoidal fuzzy number. A function, called psi $(\psi)$, is defined in the following to find the maximum between a trapezoidal fuzzy number and zero.

$$
\psi(A)=\left\{\begin{array}{l}
A \text { if } k(A)>0 \\
0 \text { if } k(A) \leq 0
\end{array}\right.
$$

where ${ }^{\delta}=(0,0,0,0)$. 


\section{Fuzzy EDAS Method}

The Evaluation based on Distance from Average Solution (EDAS) method was developed by (Keshavarz et al., 2015) for multi-criteria inventory classification. In fuzzy EDAS method, the decision-makers express the weights of the criteria and the rating of alternatives with respect to each criterion by linguistic terms. These linguistic terms are quantified by positive trapezoidal fuzzy numbers. The steps of the extended fuzzy EDAS method are presented as follows: (Keshavarz et al., 2016).

Step 1: Construct the average decision matrix $(\mathrm{X})$, shown as follows:

$$
\begin{aligned}
& X=\left[\tilde{x}_{i j}\right]_{n x m} \\
& \tilde{x}_{i j}=\frac{1}{k} \oplus_{p=1}^{k} \tilde{x}_{i j}^{p} \\
& \text { where } \tilde{x}_{i j d e n o t e s}^{p}
\end{aligned}
$$
$A_{i}(1 \leq \mathrm{i} \leq \mathrm{n})$ with respect to criterion $\mathrm{c}_{\mathrm{j}}(1 \leq \mathrm{j} \leq \mathrm{m})$ assigned by the $p^{\text {th }}$ decision-maker $(1 \leq \mathrm{p} \leq \mathrm{k})$.

Step 2: Construct the matrix of the criteria weights, shown as follows:

$$
\begin{aligned}
& W=\left[\tilde{w}_{j}\right]_{1 x m} \\
& \tilde{w}_{j}=\frac{1}{k} \oplus_{p=1}^{k} \tilde{w}_{j}^{p} \\
& \text { where } \tilde{w}_{j}^{p} \text { denotes }
\end{aligned}
$$
assigned by the $p^{\text {th }}$ decision-maker $(1 \leq \mathrm{p} \leq \mathrm{k})$.

Step 3: Construct the matrix of average solutions, shown as follows:

$$
\begin{aligned}
& A V=\left[\tilde{\omega}_{j}\right]_{1 \times m} \\
& \widetilde{a v}_{j}=\frac{1}{n} \oplus_{i=1}^{n} \tilde{x}_{i j}
\end{aligned}
$$

The elements of this matrix $a v_{\mathrm{j}}$ represent the average solutions with respect to each criterion. Therefore, the dimension of the matrix is equal to the dimension of the criteria weights matrix.

Step 4: Suppose that B is the set of beneficial criteria and $\mathrm{N}$ is the set of non-beneficial criteria. In this step, the matrices of positive distance from average (PDA) and negative distance from average (NDA) are calculated according to the type of criteria (beneficial and nonbeneficial), shown as follows:

$$
\begin{aligned}
& P D A=\left[\overrightarrow{p d a_{i j j}}\right]_{n x m} \\
& N D A=\left[\tilde{n d a_{i j}}\right]_{n \times m}
\end{aligned}
$$

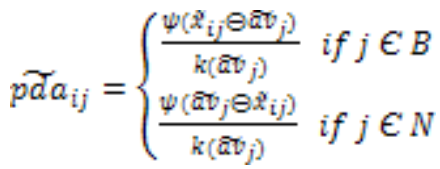

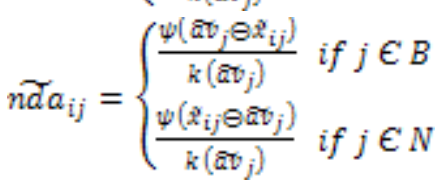

where $\hat{p d a_{i j j}}$ and $\tilde{n d a_{i j}}$ denote the positive and negative distance of performance value of $i^{\text {th }}$ alternative from the average solution in terms of $\mathrm{j}^{\text {th }}$ criterion, respectively.

Step 5: Calculate the weighted sum of positive and negative distances for all alternatives, shown as follows:

$$
\begin{aligned}
& \widetilde{p}_{i}=\Theta_{i=1}^{m}\left(\tilde{w}_{j} \otimes \overrightarrow{p d a_{i j}}\right) \\
& \tilde{s}_{i}=\oplus_{j=1}^{m}\left(\tilde{w}_{j} \otimes n d a_{i j}\right)
\end{aligned}
$$

Step 6: The normalize values of $s p_{i}$ and $s n_{i}$ for all alternatives are calculated as follows:

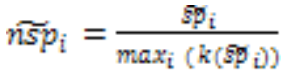

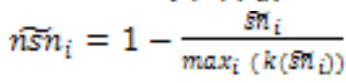

Step 7: Calculate the appraisal score $\left(\widetilde{a s}_{i}\right)$ for all alternatives, shown as follows:

$$
\widetilde{\omega s}_{i}=\frac{1}{2}\left(\tilde{n} \overrightarrow{s p}_{i} \otimes \tilde{n} \tilde{s}_{i}\right)
$$

Step 8: Rank the alternatives according to the decreasing values of appraisal scores $\left(\widetilde{a s}_{i}\right)$. In other words, the alternative with the highest appraisal score is the best choice among the candidate alternatives.

\section{The Selection of Manufacturer of PVC Carpentry}

The market is filled with manufacturers of PVC carpentry which offer various products. Through research made by decision-makers, they have selected the seven manufacturers, all located on maximal distance of $50 \mathrm{~km}$. The surface of apartment which requires the selection of the most suitable manufacturer of PVC carpentry is $67 \mathrm{~m}^{2}$ and Figure 1 shows dimensions of all the apartment surfaces in need of PVC carpentry.

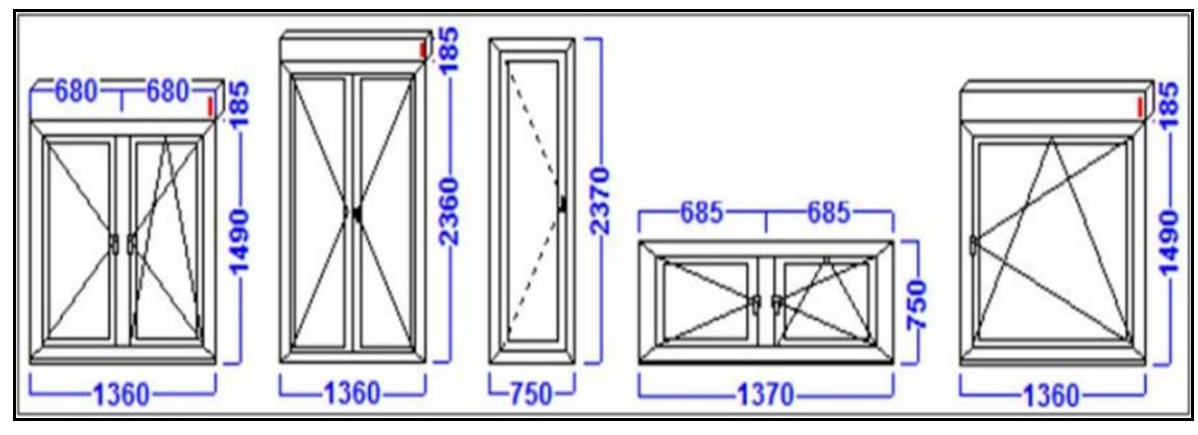

Figure 1. Dimensions of Elements Needed for Refurbishment

As it can be seen in Figure 1, according to the wish of buyers - decision-makers, there are surfaces that need carpentry together with window blinds and several surfaces without window blinds. The first window shown in Figure 1 is double-hung and needs two pieces, and it is intended to be placed in the living room. The following elements in 
Figure 1 are double-hung balcony doors for the kitchen, without blinds. The penultimate element displayed in the figure is a double-hung bathroom window without blinds and the last element is a single-hung window with blinds needed in two pieces intended for two bedrooms.

Criteria formed in this research and the base for decision-makers to make the selection of the most suitable manufacturer are: quality of product, price of product, guarantee timeframe, number of product chambers, reliability, flexibility, communication system, delivery time, payment methods, price of assembly and disassembly, manufacturer reputation, financial stability, response time and production capacity marked hereinafter as $\mathrm{C}_{1}-\mathrm{C}_{14}$, respectively. The second, the eighth, the tenth and the thirteenth criteria are expense criteria, the rest of the criteria are benefit criteria. Table 1 shows linguistic variables and trapezoidal numbers for markings of values of the criteria and importance of alternatives.

Table 1

Linguistic Terms and their Corresponding Trapezoidal Fuzzy Number

\begin{tabular}{|c|c|c|}
\hline Terms & TFN for weighting criteria & TFN for rating alternatives \\
\hline Very low (VL) & $(0,0,0.1,0.2)$ & $(0,0,1,2)$ \\
\hline Low (L) & $(0.1,0.2,0.2,0.3)$ & $(1,2,2,3)$ \\
\hline Medium low (ML) & $(0.2,0.3,0.4,0.5)$ & $(4,5,4,5)$ \\
\hline Medium (M) & $(0.4,0.5,0.5,0.6)$ & $(5,6,7,8)$ \\
\hline Medium high (MH) & $(0.5,0.6,0.7,0.8)$ & $(7,8,8,9)$ \\
\hline High (H) & $(0.7,0.8,0.8,0.9)$ & $(8,9,10,10)$ \\
\hline Very high (VH) & $(0.8,0.9,1.0,1.0)$ & $p)$ \\
\hline
\end{tabular}

Table 2

The weight of Criteria Assigned by Decision-Makers and the Matrix of Criteria Weights

\begin{tabular}{|c|c|c|c|}
\hline Criteria & $\mathbf{D M}_{\mathbf{1}}$ & $\mathbf{D M}_{\mathbf{2}}$ & $\mathbf{W}_{\mathbf{j}}$ \\
\hline $\mathrm{C}_{1}$ & $\mathrm{VH}$ & $\mathrm{VH}$ & $(0.80,0.90,1.0,1.0)$ \\
\hline $\mathrm{C}_{2}$ & $\mathrm{H}$ & $\mathrm{H}$ & $(0.70,0.80,0.80,0.90)$ \\
\hline $\mathrm{C}_{3}$ & $\mathrm{ML}$ & $\mathrm{MH}$ & $(0.35,0.45,0.55,0.65)$ \\
\hline $\mathrm{C}_{4}$ & $\mathrm{~L}$ & $\mathrm{M}$ & $(0.25,0.35,0.35,0.45)$ \\
\hline $\mathrm{C}_{5}$ & $\mathrm{VL}$ & $\mathrm{M}$ & $(0.20,0.25,0.30,0.40)$ \\
\hline $\mathrm{C}_{6}$ & $\mathrm{M}$ & $\mathrm{ML}$ & $(0.30,0.40,0.45,0.55)$ \\
\hline $\mathrm{C}_{7}$ & $\mathrm{MH}$ & $\mathrm{H}$ & $(0.60,0.70,0.75,0.85)$ \\
\hline $\mathrm{C}_{8}$ & $\mathrm{H}$ & $\mathrm{VH}$ & $(0.75,0.85,0.90,0.95)$ \\
\hline $\mathrm{C}_{9}$ & $\mathrm{MH}$ & $\mathrm{MH}$ & $(0.50,0.60,0.70,0.80)$ \\
\hline $\mathrm{C}_{10}$ & $\mathrm{~L}$ & $\mathrm{ML}$ & $(0.15,0.25,0.30,0.40)$ \\
\hline $\mathrm{C}_{11}$ & $\mathrm{M}$ & $\mathrm{M}$ & $(0.40,0.50,0.50,0.60)$ \\
\hline $\mathrm{C}_{12}$ & $\mathrm{MH}$ & $\mathrm{M}$ & $(0.45,0.55,0.60,0.70)$ \\
\hline $\mathrm{C}_{13}$ & $\mathrm{H}$ & $\mathrm{MH}$ & $(0.60,0.70,0.75,0.85)$ \\
\hline $\mathrm{C}_{14}$ & $\mathrm{M}$ & $\mathrm{M}$ & $(0.40,0.50,0.50,0.60)$ \\
\hline
\end{tabular}

As already stated, there are two decision-makers in the study, a young married couple. In Table 2, an importance of every criteria has been displayed individually toward both decision-makers applying linguistic variables from Table 1. Moreover, a weight value of the criteria reached through an equation (16) has been displayed.
Two decision-makers are perhaps not enough in group decision-making, but, in the practical example addressed in this paper, they are sufficient and only correct. The reason for this is the fact that the decision-makers are the only ones who live in that apartment and it would be very nonobjective to involve more decision-makers since needs, demands and opportunities differ from a person to person. The goal is to make a right and objective decision taking into account only relevant facts as it is the case in this paper.

It is possible to conclude from Table 2 that the most important criterion in selection of the manufacturer of PVC carpentry is criterion number one, which is product quality, then time needed for production and delivery, then price, where other criteria have a slightly lower importance. As other important criteria, we can mention communication system that is initial contact with the staff employed in the company and response time, because these two factors also play an important part in decision-making process, as the aim of the couple is to complete the works as soon as possible in order to move in.

Table 3 displays evaluation of alternatives with the use of linguistic variables of each criterion used by the decision-making couple.

Table 3

The Rating of Alternatives According to the Criteria Expressed in Linguistic Variables

\begin{tabular}{|c|c|c|c|c|c|c|c|c|c|c|c|c|c|c|c|}
\hline \multicolumn{2}{|c|}{ DM } & $\mathrm{C}_{1}$ & $\mathbf{C}_{2}$ & $\mathrm{C}_{3}$ & $\mathrm{C}_{4}$ & $\mathrm{C}_{5}$ & $\mathrm{C}_{6}$ & $\mathbf{C}_{7}$ & $\mathrm{C}_{8}$ & $\mathrm{C}_{9}$ & $\mathrm{C}_{10}$ & $\mathrm{C}_{11}$ & $\mathrm{C}_{12}$ & $\mathbf{C}_{13}$ & $\mathrm{C}_{14}$ \\
\hline \multirow{7}{*}{1} & $\mathrm{~A}_{1}$ & $\mathrm{H}$ & VL & $\mathrm{L}$ & $\mathrm{H}$ & $\mathrm{H}$ & $\mathrm{VH}$ & $\mathrm{H}$ & MH & $\mathrm{M}$ & $\mathrm{H}$ & $\mathrm{H}$ & $\mathrm{VH}$ & MH & $\mathrm{VH}$ \\
\hline & $\mathrm{A}_{2}$ & VL & $\mathrm{VH}$ & $\mathrm{L}$ & MH & MH & $\mathrm{VH}$ & $\mathrm{VH}$ & $\mathrm{H}$ & $\mathrm{M}$ & $\mathrm{H}$ & $\mathrm{L}$ & $\mathrm{H}$ & $\mathrm{H}$ & MH \\
\hline & $\mathrm{A}_{3}$ & $\mathrm{MH}$ & VL & ML & $\mathrm{H}$ & $\mathrm{VH}$ & $\mathrm{H}$ & $\mathrm{MH}$ & $\mathrm{M}$ & ML & $\mathrm{H}$ & $\mathrm{H}$ & $\mathrm{VH}$ & MH & $\mathrm{H}$ \\
\hline & $\mathrm{A}_{4}$ & $\mathrm{VH}$ & $\mathrm{VH}$ & VH & $\mathrm{VH}$ & ML & $\mathrm{MH}$ & $\mathrm{MH}$ & VH & $\mathrm{VH}$ & $\mathrm{VH}$ & $\mathrm{H}$ & $\mathrm{VH}$ & $\mathrm{H}$ & $\mathrm{VH}$ \\
\hline & $\mathrm{A}_{5}$ & $\mathrm{MH}$ & $\mathrm{M}$ & $\mathrm{VH}$ & $\mathrm{H}$ & $\mathrm{H}$ & $\mathrm{H}$ & $\mathrm{H}$ & $\mathrm{H}$ & MH & $\mathrm{M}$ & $\mathrm{H}$ & $\mathrm{H}$ & $\mathrm{H}$ & $\mathrm{H}$ \\
\hline & $\mathrm{A}_{6}$ & $\mathrm{H}$ & $\mathrm{H}$ & $\mathrm{H}$ & $\mathrm{H}$ & $\mathrm{H}$ & $\mathrm{VH}$ & $\mathrm{VH}$ & $\mathrm{MH}$ & $\mathrm{MH}$ & $\mathrm{H}$ & $\mathrm{VH}$ & $\mathrm{H}$ & $\mathrm{VH}$ & $\mathrm{VH}$ \\
\hline & $\mathrm{A}_{7}$ & $\mathrm{VH}$ & ML & MH & $\mathrm{H}$ & $\mathrm{H}$ & MH & $\mathrm{H}$ & $\mathrm{H}$ & MH & $\mathrm{H}$ & $\mathrm{VH}$ & $\mathrm{H}$ & $\mathrm{MH}$ & MH \\
\hline \multirow{7}{*}{2} & $\mathrm{~A}_{1}$ & $\mathrm{VH}$ & VL & VL & $\mathrm{H}$ & $\mathrm{H}$ & $\mathrm{H}$ & $\mathrm{MH}$ & $\mathrm{H}$ & $\mathrm{M}$ & $\mathrm{H}$ & $\mathrm{VH}$ & $\mathrm{VH}$ & $\mathrm{H}$ & $\mathrm{H}$ \\
\hline & $\mathrm{A}_{2}$ & $\mathrm{~L}$ & $\mathrm{VH}$ & $\mathrm{L}$ & $\mathrm{M}$ & $\mathrm{H}$ & $\mathrm{VH}$ & $\mathrm{VH}$ & $\mathrm{H}$ & MH & MH & ML & $\mathrm{VH}$ & $\mathrm{H}$ & $\mathrm{H}$ \\
\hline & $\mathrm{A}_{3}$ & MH & $\mathrm{L}$ & $\mathrm{M}$ & $\mathrm{H}$ & $\mathrm{H}$ & $\mathrm{H}$ & $\mathrm{H}$ & MH & $\mathrm{L}$ & $\mathrm{H}$ & $\mathrm{H}$ & $\mathrm{H}$ & MH & $\mathrm{H}$ \\
\hline & $\mathrm{A}_{4}$ & $\mathrm{VH}$ & $\mathrm{H}$ & VH & $\mathrm{VH}$ & $\mathrm{M}$ & $\mathrm{H}$ & $\mathrm{H}$ & VH & $\mathrm{VH}$ & $\mathrm{H}$ & MH & $\mathrm{H}$ & VH & $\mathrm{VH}$ \\
\hline & $\mathrm{A}_{5}$ & $\mathrm{H}$ & ML & $\mathrm{H}$ & $\mathrm{H}$ & $\mathrm{H}$ & $\mathrm{H}$ & $\mathrm{VH}$ & $\mathrm{H}$ & MH & $\mathrm{M}$ & $\mathrm{H}$ & $\mathrm{H}$ & $\mathrm{H}$ & $\mathrm{VH}$ \\
\hline & $\mathrm{A}_{6}$ & VH & MH & VH & $\mathrm{H}$ & VH & $\mathrm{H}$ & $\mathrm{VH}$ & MH & $\mathrm{H}$ & $\mathrm{H}$ & $\mathrm{H}$ & VH & $\mathrm{VH}$ & VH \\
\hline & $\mathrm{A}_{7}$ & $\mathrm{H}$ & ML & $\mathrm{MH}$ & $\mathrm{H}$ & $\mathrm{H}$ & $\mathrm{H}$ & $\mathrm{MH}$ & MH & $\mathrm{H}$ & $\mathrm{H}$ & $\mathrm{H}$ & $\mathrm{MH}$ & $\mathrm{M}$ & $\mathrm{H}$ \\
\hline
\end{tabular}


Apart from the values of the average decision matrix, Table 4 also shows the values of an average solution according to all the criteria obtained through the following expressions (17) and (18).

Then, we need to calculate positive distances (PDA) and negative distances (NDA) from the average solutions depending on the criteria type. By applying equations (21), we get the values of the positive distance (PDA) (Table 5) and (22), the values of the negative distance (NDA) from the average solution (Table 6).

The Elements of the Average Decision Matrix and the Average Solution Matrix

\begin{tabular}{|c|c|c|c|c|c|c|c|c|c|c|c|c|c|c|c|c|}
\hline & \multicolumn{4}{|c|}{ A1 } & \multicolumn{4}{|c|}{$\mathbf{A 2}$} & \multicolumn{4}{|c|}{$\mathbf{A 3}$} & \multicolumn{4}{|c|}{$\overline{\mathrm{A4}}$} \\
\hline $\mathrm{C}_{1}$ & 0.75 & 0.85 & 0.90 & 0.95 & 0.05 & 0.10 & 0.15 & 0.25 & 0.50 & 0.60 & 0.70 & 0.80 & 0.80 & 0.90 & 1.00 & 1.00 \\
\hline $\mathrm{C}_{2}$ & 0.00 & 0.00 & 0.10 & 0.20 & 0.80 & 0.90 & 1.00 & 1.00 & 0.05 & 0.10 & 0.15 & 0.25 & 0.75 & 0.85 & 0.90 & 0.95 \\
\hline $\mathrm{C}_{3}$ & 0.05 & 0.10 & 0.15 & 0.25 & 0.10 & 0.20 & 0.20 & 0.30 & 0.30 & 0.40 & 0.45 & 0.55 & 0.80 & 0.90 & 1.00 & 1.00 \\
\hline $\mathrm{C}_{4}$ & 0.70 & 0.80 & 0.80 & 0.90 & 0.45 & 0.55 & 0.60 & 0.70 & 0.70 & 0.80 & 0.80 & 0.90 & 0.80 & 0.90 & 1.00 & 1.00 \\
\hline $\mathrm{C}_{5}$ & 0.70 & 0.80 & 0.80 & 0.90 & 0.60 & 0.70 & 0.75 & 0.85 & 0.75 & 0.85 & 0.90 & 0.95 & 0.30 & 0.40 & 0.45 & 0.55 \\
\hline $\mathrm{C}_{6}$ & 0.75 & 0.85 & 0.90 & 0.95 & 0.80 & 0.90 & 1.00 & 1.00 & 0.70 & 0.80 & 0.80 & 0.90 & 0.60 & 0.70 & 0.75 & 0.85 \\
\hline $\mathrm{C}_{7}$ & 0.60 & 0.70 & 0.75 & 0.85 & 0.80 & 0.90 & 1.00 & 1.00 & 0.60 & 0.70 & 0.75 & 0.85 & 0.60 & 0.70 & 0.75 & 0.85 \\
\hline $\mathrm{C}_{8}$ & 0.60 & 0.70 & 0.75 & 0.85 & 0.70 & 0.80 & 0.80 & 0.90 & 0.45 & 0.55 & 0.60 & 0.70 & 0.80 & 0.90 & 1.00 & 1.00 \\
\hline $\mathrm{C}_{9}$ & 0.40 & 0.50 & 0.50 & 0.60 & 0.45 & 0.55 & 0.60 & 0.70 & 0.15 & 0.25 & 0.30 & 0.40 & 0.80 & 0.90 & 1.00 & 1.00 \\
\hline $\mathrm{C}_{10}$ & 0.70 & 0.80 & 0.80 & 0.90 & 0.60 & 0.70 & 0.75 & 0.85 & 0.70 & 0.80 & 0.80 & 0.90 & 0.75 & 0.85 & 0.90 & 0.95 \\
\hline $\mathrm{C}_{11}$ & 0.75 & 0.85 & 0.90 & 0.95 & 0.15 & 0.25 & 0.30 & 0.40 & 0.70 & 0.80 & 0.80 & 0.90 & 0.60 & 0.70 & 0.75 & 0.85 \\
\hline $\mathrm{C}_{12}$ & 0.80 & 0.90 & 1.00 & 1.00 & 0.75 & 0.85 & 0.90 & 0.95 & 0.75 & 0.85 & 0.90 & 0.95 & 0.75 & 0.85 & 0.90 & 0.95 \\
\hline $\mathrm{C}_{13}$ & 0.60 & 0.70 & 0.75 & 0.85 & 0.70 & 0.80 & 0.80 & 0.90 & 0.50 & 0.60 & 0.70 & 0.80 & 0.75 & 0.85 & 0.90 & 0.95 \\
\hline $\mathrm{C}_{14}$ & 0.75 & 0.85 & 0.90 & 0.95 & 0.60 & 0.70 & 0.75 & 0.85 & 0.70 & 0.80 & 0.80 & 0.90 & 0.80 & 0.90 & 1.00 & 1.00 \\
\hline & \multicolumn{4}{|c|}{ A5 } & \multicolumn{4}{|c|}{ A6 } & \multicolumn{4}{|c|}{ A7 } & \multicolumn{4}{|c|}{$\mathbf{A V}$} \\
\hline $\mathrm{C}_{1}$ & 0.60 & 0.70 & 0.75 & 0.85 & 0.75 & 0.85 & 0.90 & 0.95 & 0.75 & 0.85 & 0.90 & 0.95 & 0.60 & 0.69 & 0.76 & 0.82 \\
\hline $\mathrm{C}_{2}$ & 0.30 & 0.40 & 0.45 & 0.55 & 0.60 & 0.70 & 0.75 & 0.85 & 0.20 & 0.30 & 0.40 & 0.50 & 0.39 & 0.46 & 0.54 & 0.61 \\
\hline $\mathrm{C}_{3}$ & 0.75 & 0.85 & 0.90 & 0.95 & 0.75 & 0.85 & 0.90 & 0.95 & 0.50 & 0.60 & 0.70 & 0.80 & 0.46 & 0.56 & 0.61 & 0.69 \\
\hline $\mathrm{C}_{4}$ & 0.70 & 0.80 & 0.80 & 0.90 & 0.70 & 0.80 & 0.80 & 0.90 & 0.70 & 0.80 & 0.80 & 0.90 & 0.68 & 0.78 & 0.80 & 0.89 \\
\hline $\mathrm{C}_{5}$ & 0.70 & 0.80 & 0.80 & 0.90 & 0.75 & 0.85 & 0.90 & 0.95 & 0.70 & 0.80 & 0.80 & 0.90 & 0.64 & 0.74 & 0.77 & 0.86 \\
\hline $\mathrm{C}_{6}$ & 0.70 & 0.80 & 0.80 & 0.90 & 0.75 & 0.85 & 0.90 & 0.95 & 0.60 & 0.70 & 0.75 & 0.85 & 0.70 & 0.80 & 0.84 & 0.91 \\
\hline $\mathrm{C}_{7}$ & 0.75 & 0,85 & 0.90 & 0.95 & 0.80 & 0.90 & 1.00 & 1.00 & 0.60 & 0.70 & 0.75 & 0.85 & 0.68 & 0.78 & 0.84 & 0.91 \\
\hline $\mathrm{C}_{8}$ & 0.70 & 0.80 & 0.80 & 0.90 & 0.50 & 0.60 & 0.70 & 0.80 & 0.60 & 0.70 & 0.75 & 0.85 & 0.62 & 0.72 & 0.77 & 0.86 \\
\hline $\mathrm{C}_{9}$ & 0.50 & 0.60 & 0.70 & 0.80 & 0.60 & 0.70 & 0.75 & 0.85 & 0.60 & 0.70 & 0.75 & 0.85 & 0.50 & 0.60 & 0.66 & 0.74 \\
\hline $\mathrm{C}_{10}$ & 0.40 & 0.50 & 0.50 & 0.60 & 0.70 & 0.80 & 0.80 & 0.90 & 0.70 & 0.80 & 0.80 & 0.90 & 0.65 & 0.75 & 0.76 & 0.86 \\
\hline $\mathrm{C}_{11}$ & 0.70 & 0.80 & 0.80 & 0.90 & 0.75 & 0.85 & 0.90 & 0.95 & 0.75 & 0.85 & 0.90 & 0.95 & 0.63 & 0.73 & 0.76 & 0.84 \\
\hline $\mathrm{C}_{12}$ & 0.70 & 0.80 & 0.80 & 0.90 & 0.75 & 0.85 & 0.90 & 0.95 & 0.60 & 0.70 & 0.75 & 0.85 & 0.73 & 0.83 & 0.88 & $\begin{array}{l}0.94 \\
\end{array}$ \\
\hline $\mathrm{C}_{13}$ & 0.70 & 0.80 & 0.80 & 0.90 & 0.80 & 0.90 & 1.00 & 1.00 & 0.45 & 0.55 & 0.60 & 0.70 & 0.64 & 0.74 & 0.79 & 0.87 \\
\hline $\mathrm{C}_{14}$ & 0.75 & 0.85 & 0.90 & 0.95 & 0.80 & 0.90 & 1.00 & 1.00 & 0.60 & 0.70 & 0.75 & 0.85 & 0.71 & 0.81 & 0.87 & 0.93 \\
\hline
\end{tabular}

Table 5

Values of PDA

\begin{tabular}{|c|c|c|c|c|c|c|c|c|c|c|c|c|c|c|c|c|}
\hline & \multicolumn{4}{|c|}{ A1 } & \multicolumn{4}{|c|}{ A2 } & \multicolumn{4}{|c|}{$\overline{\mathbf{A 3}}$} & \multicolumn{4}{|c|}{ A4 } \\
\hline $\mathrm{C}_{1}$ & -0.07 & 0.09 & 0.21 & 0.35 & 0.00 & 0.00 & 0.00 & 0.00 & 0.00 & 0.00 & 0.00 & 0.00 & -0.02 & 0.14 & 0.31 & 0.40 \\
\hline $\mathrm{C}_{2}$ & 0.19 & 0.36 & 0.54 & 0.61 & 0.00 & 0.00 & 0.00 & 0.00 & 0.14 & 0.31 & 0.44 & 0.56 & 0.00 & 0.00 & 0.00 & 0.00 \\
\hline $\mathrm{C}_{3}$ & 0.00 & 0.00 & 0.00 & 0.00 & 0.00 & 0.00 & 0.00 & 0.00 & 0.00 & 0.00 & 0.00 & 0.00 & 0.11 & 0.29 & 0.44 & 0.54 \\
\hline $\mathrm{C}_{4}$ & -0.19 & 0.00 & 0.02 & 0.22 & 0.00 & 0.00 & 0.00 & 0.00 & -0.19 & 0.00 & 0.02 & 0.22 & -0.09 & 0.10 & 0.22 & 0.32 \\
\hline $\mathrm{C}_{5}$ & -0.16 & 0.03 & 0.06 & 0.26 & 0.00 & 0.00 & 0.00 & 0.00 & -0.11 & 0.08 & 0.16 & 0.31 & 0.00 & 0.00 & 0.00 & 0.00 \\
\hline $\mathrm{C}_{6}$ & -0.16 & 0.01 & 0.10 & 0.25 & -0.11 & 0.06 & 0.20 & 0.30 & 0.00 & 0.00 & 0.00 & 0.00 & 0.00 & 0.00 & 0.00 & 0.00 \\
\hline $\mathrm{C}_{7}$ & 0.00 & 0.00 & 0.00 & 0.00 & -0.11 & 0.06 & 0.22 & 0.32 & 0.00 & 0.00 & 0.00 & 0.00 & 0.00 & 0.00 & 0.00 & 0.00 \\
\hline $\mathrm{C}_{8}$ & -0.23 & -0.03 & 0.07 & 0.26 & 0.00 & 0.00 & 0.00 & 0.00 & -0.08 & 0.12 & 0.22 & 0.41 & 0.00 & 0.00 & 0.00 & 0.00 \\
\hline $\mathrm{C}_{9}$ & 0.00 & 0.00 & 0.00 & 0.00 & 0.00 & 0.00 & 0.00 & 0.00 & 0.00 & 0.00 & 0.00 & 0.00 & 0.06 & 0.24 & 0.40 & 0.50 \\
\hline $\mathrm{C}_{10}$ & 0.00 & 0.00 & 0.00 & 0.00 & -0.20 & 0.00 & 0.06 & 0.26 & 0.00 & 0.00 & 0.00 & 0.00 & 0.00 & 0.00 & 0.00 & 0.00 \\
\hline $\mathrm{C}_{11}$ & -0.09 & 0.09 & 0.17 & 0.32 & 0.00 & 0.00 & 0.00 & 0.00 & -0.14 & 0.04 & 0.07 & 0.27 & 0.00 & 0.00 & 0.00 & 0.00 \\
\hline $\mathrm{C}_{12}$ & -0.14 & 0.02 & 0.17 & 0.27 & -0.19 & -0.03 & 0.07 & 0.22 & -0.19 & -0.03 & 0.07 & 0.22 & -0.19 & -0.03 & 0.07 & 0.22 \\
\hline $\mathrm{C}_{13}$ & -0.21 & -0.01 & 0.09 & 0.27 & 0.00 & 0.00 & 0.00 & 0.00 & -0.16 & 0.04 & 0.19 & 0.37 & 0.00 & 0.00 & 0.00 & 0.00 \\
\hline $\mathrm{C}_{14}$ & -0.18 & $\begin{array}{c}-0.02 \\
\end{array}$ & 0.09 & 0.24 & 0.00 & 0.00 & 0.00 & 0.00 & 0.00 & 0.00 & 0.00 & 0.00 & -0.13 & 0.03 & 0.19 & 0.29 \\
\hline & \multicolumn{4}{|c|}{ A5 } & \multicolumn{4}{|c|}{ A6 } & \multicolumn{4}{|c|}{ A7 } & & & & \\
\hline $\mathrm{C}_{1}$ & -0.22 & -0.06 & 0,06 & 0.25 & -0.07 & 0.09 & 0.21 & 0.35 & -0.07 & 0.09 & 0.21 & 0.35 & & & & \\
\hline $\mathrm{C}_{2}$ & -0.16 & 0.01 & 0,14 & 0.31 & 0.00 & 0.00 & 0.00 & 0.00 & -0.11 & 0.06 & 0.24 & 0.41 & & & & \\
\hline $\mathrm{C}_{3}$ & 0.06 & 0,24 & 0.34 & 0.49 & 0.06 & 0.24 & 0.34 & 0.49 & -0.19 & -0.01 & 0.14 & 0.34 & & & & \\
\hline $\mathrm{C}_{4}$ & -0.19 & 0,00 & 0.02 & 0.22 & -0.19 & 0.00 & 0.02 & 0.22 & -0.19 & 0.00 & 0.02 & 0.22 & & & & \\
\hline $\mathrm{C}_{5}$ & -0.16 & 0.03 & 0.06 & 0.26 & -0.11 & 0.08 & 0.16 & 0.31 & -016 & 0.03 & 0.06 & 0.26 & & & & \\
\hline $\mathrm{C}_{6}$ & 0.00 & 0.00 & 0.00 & 0.00 & -0.16 & 0.01 & 0.10 & 0.25 & 0.00 & 0.00 & 0.00 & 0.00 & & & & \\
\hline $\mathrm{C}_{7}$ & -0.16 & 0.01 & 0.12 & 0.27 & -0.11 & 0.06 & 0.22 & 0.32 & 0.00 & 0.00 & 0.00 & 0.00 & & & & \\
\hline $\mathrm{C}_{8}$ & 0.00 & 0.00 & 0.00 & 0.00 & -0.18 & 0.02 & 0.17 & 0.36 & -0.23 & -0.03 & 0.07 & 0.26 & & & & \\
\hline $\mathrm{C}_{9}$ & -0.24 & -0.06 & 0.10 & 0.30 & -0.14 & 0.04 & 0.15 & 0.35 & -0.14 & 0.04 & 0.15 & 0.35 & & & & \\
\hline $\mathrm{C}_{10}$ & 0.05 & 0.25 & 0.26 & 0.46 & 0.00 & 0.00 & 0.00 & 0.00 & 0.00 & 0.00 & 0.00 & 0.00 & & & & \\
\hline $\mathrm{C}_{11}$ & -0.14 & 0.04 & 0.07 & 0.27 & -0.09 & 0.09 & 0.17 & 0.32 & -0.09 & 0.09 & 0.17 & 0.32 & & & & \\
\hline $\mathrm{C}_{12}$ & 000 & 0.00 & 0.00 & 0.00 & -0.19 & -0.03 & 0.07 & 0.22 & 0.00 & 0.00 & 0.00 & 0.00 & & & & \\
\hline $\mathrm{C}_{13}$ & 0.00 & 0.00 & 0.00 & 0.00 & 0.00 & 0.00 & 0.00 & 0.00 & -0.06 & 0.14 & 0.24 & 0.42 & & & & \\
\hline $\mathrm{C}_{14}$ & -0.18 & -0.02 & 0.09 & 0.24 & -0.13 & 0.03 & 0.19 & 0.29 & 0.00 & 0.00 & 0.00 & 0.00 & & & & \\
\hline
\end{tabular}


Values of NDA

Table 6

\begin{tabular}{|c|c|c|c|c|c|c|c|c|c|c|c|c|c|c|c|c|}
\hline & \multicolumn{4}{|c|}{ A1 } & \multicolumn{4}{|c|}{ A2 } & \multicolumn{4}{|c|}{$\mathbf{A 3}$} & \multicolumn{4}{|c|}{ A4 } \\
\hline $\mathrm{C}_{1}$ & 0.00 & 0.00 & 0.00 & 0.00 & 0.35 & 0.54 & 0.66 & 0.77 & -0.20 & -0.01 & 0.16 & 0.32 & 0.00 & 0.00 & 0.00 & 0.00 \\
\hline $\mathrm{C}_{2}$ & 0.00 & 0.00 & 0.00 & 0.00 & $\begin{array}{l}0.19 \\
\end{array}$ & 0.36 & 0.54 & 0.61 & 0.00 & 0.00 & 0.00 & 0.00 & 0.14 & 0.31 & 044 & 0.56 \\
\hline $\mathrm{C}_{3}$ & 0.21 & 0.41 & 0.51 & 0.64 & 0.16 & 0.36 & 0.41 & 0.59 & -0.09 & 0.11 & 0.21 & 0.39 & 0.00 & 0.00 & 0.00 & 0.00 \\
\hline $\mathrm{C}_{4}$ & 0.00 & 0.00 & 0.00 & 0.00 & $\begin{array}{l}-0.02 \\
\end{array}$ & 0.18 & 0.25 & 0.44 & 0.00 & 0.00 & 0.00 & 0.00 & 0.00 & 0.00 & 0.00 & 0.00 \\
\hline $\mathrm{C}_{5}$ & 0.00 & 0.00 & 0.00 & 0.00 & -0.21 & -0.01 & 0.07 & 0.26 & 0.00 & 0.00 & 0.00 & 0.00 & 0.09 & 0.29 & 0.37 & 0.56 \\
\hline $\mathrm{C}_{6}$ & 0.00 & 0.00 & 0.00 & 0.00 & 0.00 & 0.00 & 0.00 & 0.00 & -0.20 & 0.00 & 0.04 & 0.21 & -0.15 & 0.05 & 0.14 & 0.31 \\
\hline $\mathrm{C}_{7}$ & -0.17 & 0.03 & 0.14 & 031 & 000 & 0.00 & 0.00 & 0.00 & -0.17 & 0.03 & 0.14 & 0.31 & -0.17 & 0.03 & 0.14 & 0.31 \\
\hline $\mathrm{C}_{8}$ & 0.00 & 0.00 & 0.00 & 0.00 & -0.16 & 0.03 & 0.08 & 0.28 & 0.00 & 0.00 & 0.00 & 0.00 & -0.06 & $\begin{array}{l}0.13 \\
\end{array}$ & 0.28 & 0.38 \\
\hline $\mathrm{C}_{9}$ & -0.10 & 0.10 & 0.16 & 0.34 & -0.20 & 0.00 & 0.11 & 0.29 & 0.10 & 0.30 & 0.41 & 0.59 & 0.00 & 0.00 & 0.00 & 0.00 \\
\hline $\mathrm{C}_{10}$ & -0.16 & 0.04 & 0.05 & 0.25 & 000 & 0.00 & 0.00 & 0.00 & -0.16 & 0.04 & 0.05 & 0.25 & -0.11 & 0.09 & 0.15 & 0.30 \\
\hline $\mathrm{C}_{11}$ & 0.00 & 0.00 & 0.00 & 0.00 & 0.23 & 0.43 & 0.51 & 0.69 & 0.00 & 0.00 & 0.00 & 0.00 & -0.22 & -0.02 & 0.06 & 0.24 \\
\hline $\mathrm{C}_{12}$ & 0.00 & 0.00 & 0.00 & 0.00 & 000 & 0.00 & 0.00 & 0.00 & 0.00 & 0.00 & 0.00 & 0.00 & 0.00 & 0.00 & 0.00 & 0.00 \\
\hline $\mathrm{C}_{13}$ & 0.00 & 0.00 & 0.00 & 0.00 & $\begin{array}{l}-017 \\
\end{array}$ & 0.01 & 0.06 & 0.26 & 0.00 & 0.00 & 0.00 & 0.00 & -0.12 & 0.06 & 0.16 & 0.31 \\
\hline \multirow[t]{2}{*}{$\mathrm{C}_{14}$} & 0.00 & 0.00 & 0.00 & 0.00 & -0.14 & 0.06 & 0.17 & 0.33 & -0.19 & 0.01 & 0.07 & 0.23 & 0.00 & 0.00 & 0.00 & 0.00 \\
\hline & \multicolumn{4}{|c|}{ A5 } & \multicolumn{4}{|c|}{ A6 } & \multicolumn{4}{|c|}{ A7 } & & & & \\
\hline $\mathrm{C}_{1}$ & 0.00 & 0.00 & 0.00 & 0.00 & 0.00 & 0.00 & 0.00 & 0.00 & 0.00 & 0.00 & 0.00 & 0.00 & & & & \\
\hline $\mathrm{C}_{2}$ & 0.00 & 0.00 & 0.00 & 0.00 & -0.01 & 0.16 & 0.29 & 0.46 & 0.00 & 0.00 & 0.00 & 0.00 & & & & \\
\hline $\mathrm{C}_{3}$ & 0.00 & 0.00 & 0.00 & 0.00 & 0.00 & 0.00 & 0.00 & 0.00 & 0.00 & 0.00 & 0.00 & 0.00 & & & & \\
\hline $\mathrm{C}_{4}$ & 0.00 & 0.00 & 0.00 & 0.00 & 0.00 & 0.00 & 0.00 & 0.00 & 0.00 & 0.00 & 0.00 & 0.00 & & & & \\
\hline $\mathrm{C}_{5}$ & 0.00 & 0.00 & 0.00 & 0.00 & 0.00 & 0.00 & 0.00 & 0.00 & 0.00 & 0.00 & 0.00 & 0.00 & & & & \\
\hline $\mathrm{C}_{6}$ & -0.20 & 0.00 & 0.04 & 0.21 & 0.00 & 0.00 & 0.00 & 0.00 & -0.15 & 0.05 & 0.14 & 0.31 & & & & \\
\hline $\mathrm{C}_{7}$ & 0.00 & 0.00 & 0.00 & 0.00 & 0.00 & 0.00 & 0.00 & 0.00 & -0.17 & 0.03 & 0.14 & 0.31 & & & & \\
\hline $\mathrm{C}_{8}$ & -0.16 & 0.03 & 0.08 & 0.28 & 0.00 & 0.00 & 0.00 & 0.00 & 0.00 & 0.00 & 0.00 & 0.00 & & & & \\
\hline $\mathrm{C}_{9}$ & 000 & 0.00 & 0.00 & 0.00 & 0.00 & 0.00 & 0.00 & 0.00 & 0.00 & 0.00 & 0.00 & 0.00 & & & & \\
\hline $\mathrm{C}_{10}$ & 0.00 & 0.00 & 0.00 & 0.00 & -0.16 & 0.04 & 0.05 & 0.25 & -0.16 & 0.04 & 0.05 & 0.25 & & & & \\
\hline $\mathrm{C}_{11}$ & 0.00 & 0.00 & 0.00 & 0.00 & 0.00 & 0.00 & 0.00 & 0.00 & 0.00 & 0.00 & 0.00 & 0.00 & & & & \\
\hline $\mathrm{C}_{12}$ & -0.17 & 0.03 & 0.08 & 0.24 & 0.00 & 0.00 & 0.00 & 0.00 & -0.12 & 0.08 & 0.18 & 0.34 & & & & \\
\hline $\mathrm{C}_{13}$ & -0.17 & 0.01 & 0.06 & 0.26 & -0.07 & 0.11 & 0.26 & 0.36 & 0.00 & 0.00 & 0.00 & 0.00 & & & & \\
\hline $\mathrm{C}_{14}$ & 0.00 & 0.00 & 0.00 & 0.00 & 0.00 & 0.00 & 0.00 & 0.00 & -0.14 & 0.06 & $\begin{array}{l}0.17 \\
\end{array}$ & 0.33 & & & & \\
\hline
\end{tabular}

In order to obtain the values shown in the table below, it is necessary first to apply step 5 of fuzzy EDAS method, i.e. the equations (23) and (24), and this represents the sum of weighted matrix for positive and negative distance for all alternatives. Furthermore, it is necessary to normalize previous values by using step 6, i.e. equations (25) and (26). Finally, it is necessary to calculate the assessment of the results by using equation (27) (Table 7).

Table 7

The Weighted Sum of Distances, their Normalized Values and the Appraisal Scores

\begin{tabular}{|c|c|c|c|}
\hline & $\hat{s p_{1}}$ & $\hat{s n}_{1}$ & $\overrightarrow{n s p_{1}}$ \\
\hline $\mathrm{A}_{1}$ & $(-0.52,0.40,1.07,2.23)$ & $(-0.10,0.27,0.52,1.05)$ & $(-0.62,0.47,1.27,2.64)$ \\
\hline $\mathrm{A}_{2}$ & $(-0.21,0.05,0.32,0.68)$ & $(0.14,1.28,1.95,3.33)$ & $(-0.25,0.06,0.38,0.81)$ \\
\hline $\mathrm{A}_{3}$ & $(-0.27,0.41,0.83,1.75)$ & $(-0.40,0.26,0.74,1.65)$ & $(-0.32,0.48,0.98,2.07)$ \\
\hline $\mathrm{A}_{4}$ & $(-0.11,0.44,1.04,1.62)$ & $(-0.25,0.52,1.08,2.04)$ & $(-0.12,0.52,1.24,1.92)$ \\
\hline $\mathrm{A}_{5}$ & $(-0.68,0.11,0.70,2.01)$ & $(-0.36,0.05,0.18,0.76)$ & $(-0.81,0.13,0.83,2.38)$ \\
\hline $\mathrm{A}_{6}$ & $(-0.59,0.34,1.14,2.43)$ & $(-0.08,0.22,0.44,0.82)$ & $(-0.70,0.40,1.35,2.87)$ \\
\hline $\mathrm{A}_{7}$ & $(-0.59,0.28,0.94,2.22)$ & $(-0.28,0.12,0.38,0.95)$ & $(-0.70,0.33,1.11,2.63)$ \\
\hline & $\overline{n s n_{I}}$ & $\widehat{a s_{I}}$ & $\mathbf{k}\left(\widehat{a S^{2}}\right)$ \\
\hline $\mathrm{A}_{1}$ & $(-0.06,0.16,0.30,0.62)$ & $(-0.34,0.32,0.79,1.63)$ & 0.61 \\
\hline $\mathrm{A}_{2}$ & $(0.08,0.76,1.16,1.97)$ & $(-0.08,0.41,0.77,1.39)$ & 0.63 \\
\hline $\mathrm{A}_{3}$ & $(-0.24,0.15,0.44,0.98)$ & $(-0.28,0.32,0.71,1.53)$ & 0.58 \\
\hline $\mathrm{A}_{4}$ & $(-0.15,0.31,0.64,1.21)$ & $(-0.14,0.41,0.94,1.56)$ & 0.70 \\
\hline $\mathrm{A}_{5}$ & $(-0.21,0.03,0.11,0.45)$ & $(-0.51,0.08,0.47,1.41)$ & 0.38 \\
\hline $\mathrm{A}_{6}$ & $(-0.05,0.13,0.26,0.49)$ & $(-0.37,0.26,0.81,1.68)$ & 0.60 \\
\hline $\mathrm{A}_{7}$ & $(-0.17,0.07,0.22,0.56)$ & $(-0.43,0.20,0.67,1.59)$ & 0.52 \\
\hline
\end{tabular}

Characteristics of the selected manufacturer are as follows: PVC positions are made of the German sixchamber Inoutic PVC profile of Prestige system with three grey seals, depth of construction is $76 \mathrm{~mm}$, white colored with $1.5 \mathrm{~mm}$ reinforcement, dimensions of window frame are $76 / 85 \mathrm{~mm}$ and blinds of $84 \mathrm{~mm}$ in height, window blinds made of PVC system INOUTIC PROTEX with aluminum cover, box dimensions of $205 \times 185 \mathrm{~mm}$, Frame Roto NT, glass: IZO Flot 24 mm thick $(4+16+4)$

\section{Sensitivity Analysis and Discussion}

After obtaining the results, it is necessary to conduct a sensitivity analysis in order to ensure whether the selected solution is in fact optimal and in which frame changes affect the final ranking of alternatives, i.e. to verify the model stability. Therefore, an experiment with 14 sets where one criterion has the highest value and one has the lowest value in each set has been made. For example, set 1 indicates that the first criterion has the value of 0.01 , in the 
second set 0.02 , in the third set 0.03 , and so on until the last set with the value of 0.14 . In the same way, the other sets are formed as it can be seen in Figure 2 which represents all sets and values of each criterion in the set.

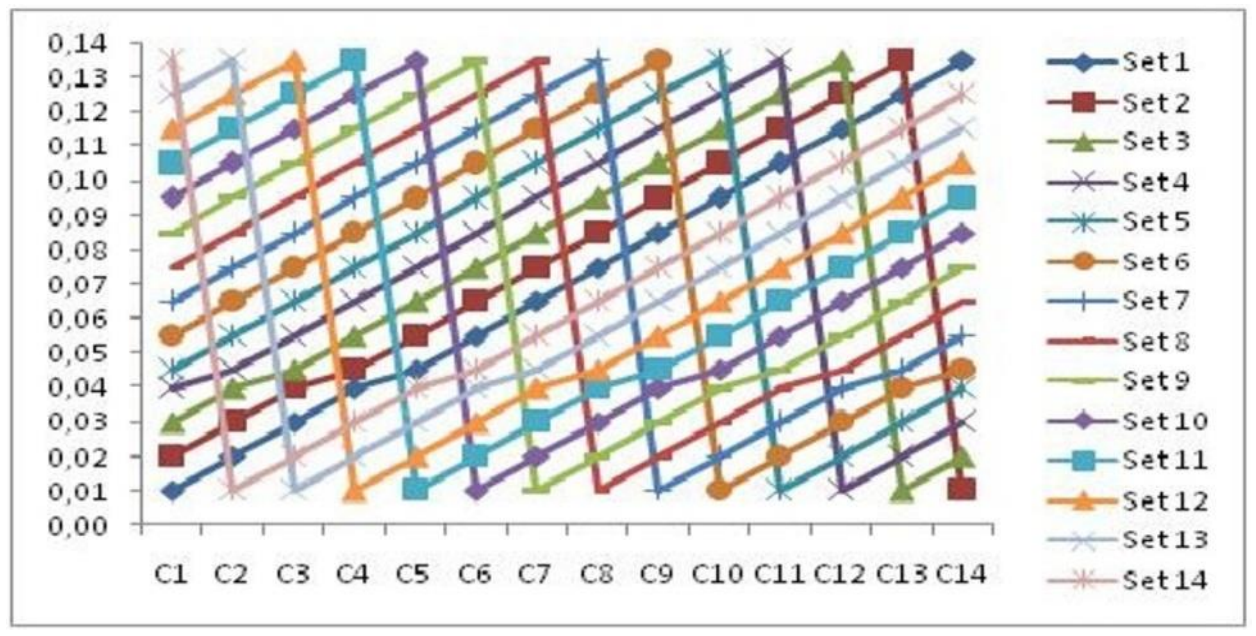

Figure 2. Criteria Values in Simulated Sets

Figure 3 displays values of all the manufacturers in all formed sets in sensitivity analysis, where it can be noticed that in the fifth set the value of manufacturer number four is the highest when the tenth criterion is with the highest value, and the eleventh criterion is with the lowest value reaching overall value of 0.94 , and, in that set, the selected manufacturer is in significant lead in comparison to other manufacturers. A slightly smaller advantage in comparison to the fifth set, the manufacturer number four has in the sixth set. In the same set, the manufacturer number five is with the lowest value of 0.30 .

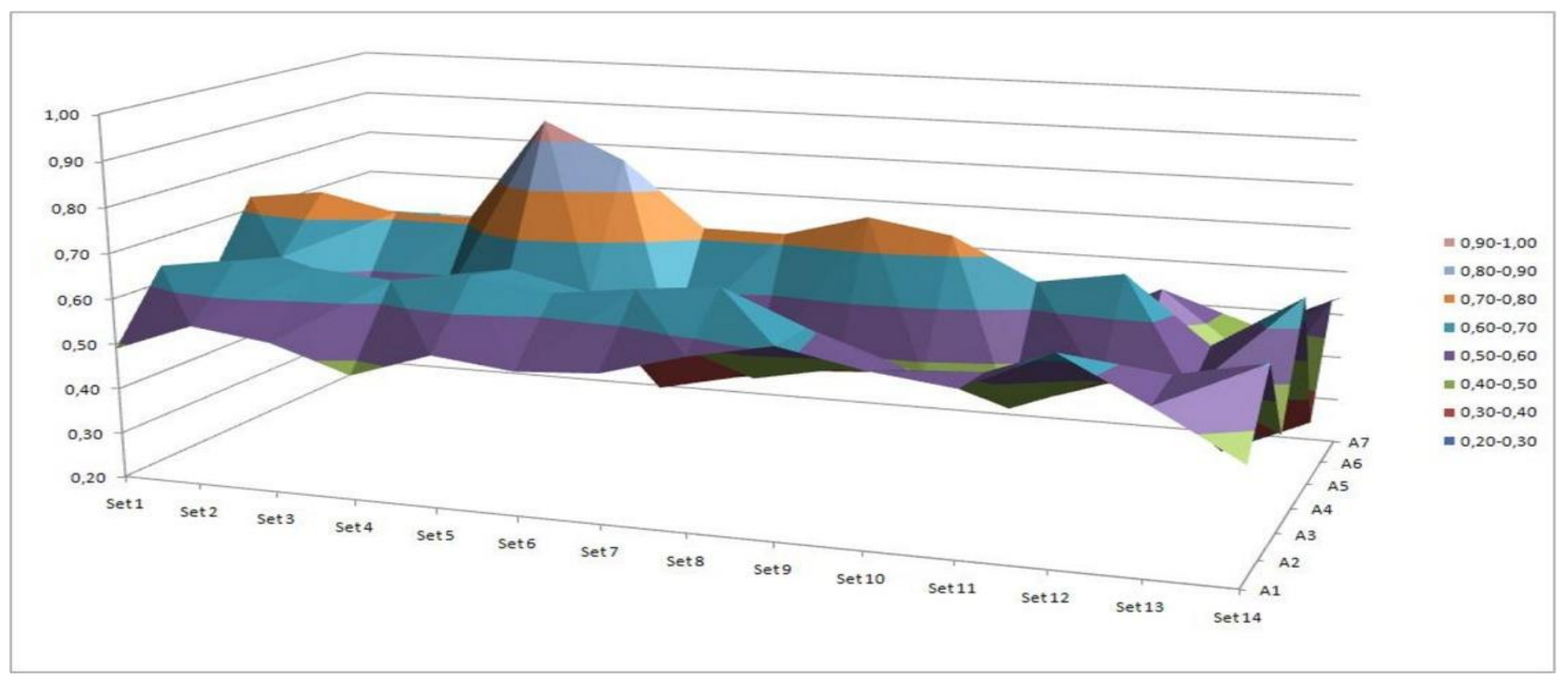

Figure 3. Values of Alternatives in Sensitivity Analysis

Figure 4 displays ranking of the manufacturers in different sets where it can be clearly seen that the selected manufacturer number four is the best solution in 13 of total of 14 sets, where the manufacturer number five has the lowest value in most cases, even in 11 sets, where in the other three sets the manufacturer number four is in the penultimate, sixth place. The largest influence on the single change in the ranking of the manufacturer number four has set 13 where the criterion of financial stability has the highest value. In other changes of the criteria weighting, the mentioned alternative remains in the first position. Manufacturer number one is more vulnerable to changing the weights of the criteria, so its position varies from the second to sixth place. Alternative number two is most commonly ranked as second, i.e. in nine sets, while by increasing the importance of criterion 12 , it is in the first position. In addition, it is twice in the third and fourth position. In the half of the sets, manufacturer number three is in the fifth position, four times in the fourth position, twice in the high third place and once in the sixth position. For alternative number six, it can be said to maintain the stability in relation to criterion weighting, as it is in the third and fourth position with the exception of set 13 when it is in the sixth position. Alternative number the seven changes its position from the fourth to the last position. 


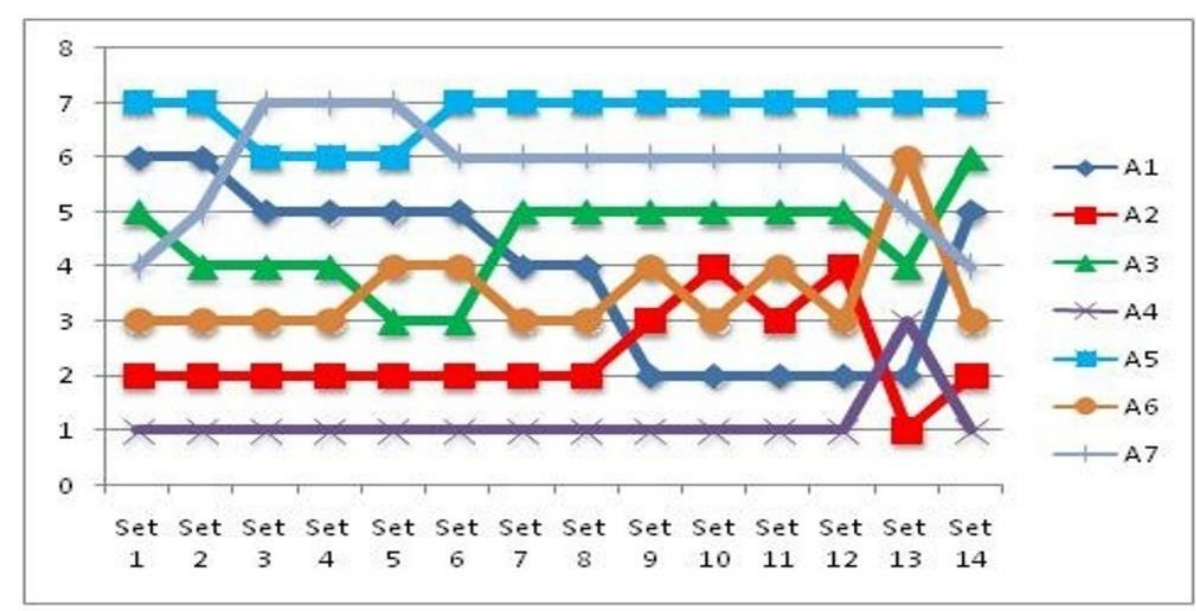

Figure 4. Ranking of Manufacturers in Sensitivity Analysis

Based on the conducted sensitivity analysis, it can be concluded that the results are stable and that, in fact, manufacturer number four is the best solution for the apartment refurbishment when it comes to installment of outdoor carpentry.

The limitations and weaknesses of the applied model are in relation to its narrow application capability. The model is defined and set up on the basis of the specific case and for any other problem it should be significantly modified.

\section{Conclusions}

Research made in this paper shows applicability of methods of multi-criteria analysis in making everyday decisions which can be of remarkable importance to every individual. Solving the problem the selection of manufacturer of PVC carpentry has used all relevant criteria which can influence the final decision. The aim was to obtain the most suitable offer, which means high quality, price as low as possible, short delivery times, quick response, possibility of monthly payments, guarantees along with reliability, but also other relevant facts which may influence the decision have not been neglected and, therefore, other criteria have also been used in order to make the decision as best as possible. When the final decision has been made on the basis of the results obtained, it can be said without hesitation that manufacturer number four represents the best solution, as it fulfills all previously mentioned criteria to a large extent. Satisfaction with the selected manufacturer who completed contracted works is on a very high quality level for both decisionmakers.

The contribution of this paper is mainly in making a real-life decision which has a great influence on the quality of life. Moreover, the model provides objective aggregation of DM decisions and takes into account subjectivity and uncertainty present in group decision-making.

When it comes to future research which will deal with the same or similar problems, it is possible to apply some of other methods of multi-criteria analysis or a combination of two methods where one is applied to reach importance of the criteria, and other for ranking of the criteria. Furthermore, it is possible to have a larger number of decision-makers, depending on the specificity of the problem and how many people are involved in solving it.

\section{References}

Akhavan, P., Barak, S., Maghsoudlou, H., \& Antucheviciene, J. (2015). FQSPM-SWOT for strategic alliance planning and partner selection; case study in a holding car manufacturer company. Technological and Economic Development of Economy, 21(2), 165-185. https://doi.org/10.3846/20294913.2014.965240

Akkaya, G., Turanoglu, B., \& Oztas, S. (2015). An integrated fuzzy AHP and fuzzy MOORA approach to the problem of industrial engineering sector choosing. Expert Systems with Applications, 42(24), 9565-9573. https://doi.org/10.1016/j.eswa.2015.07.061

Bojanic, D., Kovac, M., Bojanic, M., \& Ristic, V. (2018). Multi-criteria decision making in defensive operation of guided antitank missile battery: An example of hybrid model fuzzy AHP-MABAC. Decision Making: Applications in Management and Engineering, 1(1), 51-66. https://doi.org/10.31181/dmame180151b

Bucoń, R., \& Sobotka, A. (2015). Decision-making model for choosing residential building repair variants. Journal of Civil Engineering and Management, 21(7), 893-901. https://doi.org/10.3846/13923730.2014.895411

Chen, N., Xu, Z., \& Xia, M. (2015). The ELECTRE I Multi-Criteria Decision-Making Method Based on Hesitant Fuzzy Sets. International Journal of Information Technology \& Decision Making, 14(3), 621-657. https://doi.org/10.1142/S0219622014500187 
Chen, S. J., \& Hwang, C. L. (1992). Fuzzy Multiple Attribute Decision Making: Methods and Applications, Berlin Heidelberg: Springer. https://doi.org/10.1007/978-3-642-46768-4

Govindan, K., Rajendran, S., Sarkis, J., \& Murugesan, P. (2015). Multi criteria decision making approaches for green supplier evaluation and selection: a literature review. Journal of Cleaner Production, 98, 66-83. https://doi.org/10.1016/j.jclepro.2013.06.046

Gul, M., Celik, E., Aydin, N., Gumus, A. T., \& Guneri, A. F. (2016). A state of the art literature review of VIKOR and its fuzzy extensions on applications. Applied Soft Computing, 46, 60-89. https://doi.org/10.1016/j.asoc.2016.04.040

Jelokhani-Niaraki, M., \& Malczewski, J. (2015). A group multicriteria spatial decision support system for parking site selection problem: A case study. Land Use Policy, 42, 492-508. https://doi.org/10.1016/j.landusepol.2014.09.003

Kahraman, C., Keshavarz Ghorabaee, M., Zavadskas, E. K., Cevik Onar, S., Yazdani, M., \& Oztaysi, B. (2017). Intuitionistic fuzzy EDAS method: an application to solid waste disposal site selection. Journal of Environmental Engineering and Landscape Management, 25(1), 1-12. https://doi.org/10.3846/16486897.2017.1281139

Keshavarz Ghorabaee, M. K, Zavadskas, E. K., Olfat, L., \& Turskis, Z. (2015). Multi-Criteria Inventory Classification Using a New Method of Evaluation Based on Distance from Average Solution (EDAS). Informatica, 26(3), $435-451$. https://doi.org/10.15388/Informatica.2015.57

Keshavarz Ghorabaee, M. K., Amiri, M., Sadaghiani, J. S., \& Goodarzi, G. H. (2014). Multiple criteria group decision-making for supplier selection based on COPRAS method with interval type-2 fuzzy sets. The International Journal of Advanced Manufacturing Technology, 75(5-/), 1115-1130. https://doi.org/10.1007/s00170-014-6142-7

Keshavarz Ghorabaee, M. K., Zavadskas, E. K., Amiri, M., \& Turskis, Z. (2016). Extended EDAS Method for Fuzzy Multicriteria Decision-making: An Application to Supplier Selection. International Journal of Computers Communications \& Control, 11(3), 358-371. https://doi.org/10.15837/ijccc.2016.3.2557

Keshavarz Ghorabaee, M., Amiri, M., Zavadskas, E. K., \& Antucheviciene, J. (2018). A new hybrid fuzzy MCDM approach for evaluation of construction equipment with sustainability considerations. Archives of Civil and Mechanical Engineering, 18(1), 32-49. https://doi.org/10.1016/j.acme.2017.04.011

Keshavarz Ghorabaee, M., Amiri, M., Zavadskas, E. K., Turskis, Z., \& Antucheviciene, J. (2017). Stochastic EDAS method for multi-criteria decision-making with normally distributed data. Journal of Intelligent \& Fuzzy Systems, 33(3), 1627-1638. https://doi.org/10.3233/JIFS-17184

Macharis, C., \& Bernardini, A. (2015). Reviewing the use of Multi-Criteria Decision Analysis for the evaluation of transport projects: Time for a multi-actor approach. Transport Policy, 37, 177-186. https://doi.org/10.1016/j.tranpol.2014.11.002

Marcinkowska, E., Gawron, K., \& Rejment, M. (2015). Technical and economic aspects of revitalization of down-town tenement-houses in Wrocław. Journal of Civil Engineering and Management, 21(8), 1036-1045. https://doi.org/10.3846/13923730.2015.1027259

Mardani, A., Jusoh, A., \& Zavadskas, E. K. (2015). Multiple criteria decision-making techniques and their applications-a review of the literature from 2000 to 2014. Economic Research-Ekonomska Istrazivanja, $28(1), 516-571$. https://doi.org/10.1080/1331677X.2015.1075139

Medineckiene, M., Zavadskas, E. K., Bjork, F., \& Turskis, Z. (2015). Multi-criteria decision-making system for sustainable building assessment/certification. Archives of Civil and Mechanical Engineering, 15(1), 11-18. https://doi.org/10.1016/j.acme.2014.09.001

Morselli, A. (2015). The decision-making process between convention and cognition. Economics and Sociology, 8(1), $205-221$. https://doi.org/10.14254/2071-789x.2015/8-1/16

Motuziene, V., Rogoza, A., Lapinskiene, V., \& Vilutiene, T. (2016). Construction solutions for energy efficient single-family house based on its life cycle multi-criteria analysis: a case study. Journal of Cleaner Production, 112, 532-541. https://doi.org/10.1016/j.jclepro.2015.08.103

Nuuter, T., Lill, I., \& Tupenaite, L. (2015). Comparison of housing market sustainability in European countries based on multiple criteria assessment. Land Use Policy, 42, 642-651. https://doi.org/10.1016/j.landusepol.2014.09.022

Olcer, A. Y., \& Odabasi, A. Y. (2005). A new fuzzy multiple attributive group decision making methodology and its application to propulsion/manoeuvring system selection problem. European Journal of Operational Research, 166(1), 93-114. https://doi.org/10.1016/j.ejor.2004.02.010

Pamucar, D., \& Cirovic, G. (2015). The selection of transport and handling resources in logistics centers using Multi-Attributive Border Approximation area Comparison (MABAC). Expert Systems with Applications, 42(6), 3016-3028. https://doi.org/10.1016/j.eswa.2014.11.057

Pushkina, J., Jansons, V., \& Didenko, K. (2015). Applying Multi-Criteria Analysis Methods for Fire Risk Assessment. Safety of Technogenic Environment, 7(1), 42-47. https://doi.org/10.1515/ste-2015-0006 
Rikalovic, A., Cosic, I., \& Lazarevic, D. (2014). GIS based multi-criteria analysis for industrial site selection. Procedia Engineering, 69, 1054-1063. https://doi.org/10.1016/j.proeng.2014.03.090

Soltani, A., Hewage, K., Reza, B., \& Sadiq, R. (2015). Multiple stakeholders in multi-criteria decision-making in the context of municipal solid waste management: a review. Waste Management, 35, 318-328. https://doi.org/10.1016/j.wasman.2014.09.010

Stanujkic, D., Zavadskas, E. K., Ghorabaee, M. K., \& Turskis, Z. (2017). An extension of the EDAS method based on the use of interval grey numbers. Studies in Informatics and Control, 26(1), 5-12. https://doi.org/10.24846/v26i1y201701

Stevic, Z., Pamucar, D., Kazimieras Zavadskas, E., Cirovic, G., \& Prentkovskis, O. (2017a). The Selection of Wagons for the Internal Transport of a Logistics Company: A Novel Approach Based on Rough BWM and Rough SAW Methods. Symmetry, 9(11), 264. https://doi.org/10.3390/sym9110264

Stevic, Z., Pamucar, D., Vasiljevic, M., Stojic, G., \& Korica, S. (2017b). Novel Integrated Multi-Criteria Model for Supplier Selection: Case Study Construction Company. Symmetry, 9(11), 279. https://doi.org/10.3390/sym9110279

Stevic, Z., Tanackov, I., Vasiljevic, M., Novarlic, B., \& Stojic, G. (2016a). An integrated fuzzy AHP and TOPSIS model for supplier evaluation. Serbian Journal of Management, 11(1), 15-27.

Stevic, Z., Tanackov, I., Vasiljevic, M., Veskovic, S. (2016b) Evaluation in logistics using combined AHP and EDAS method. In Proceedings of the XLIII International Symposium on Operational Research, Belgrade, Serbia, 20-23 September pp. 309-313

Streimikiene, D., Sliogeriene, J., \& Turskis, Z. (2016). Multi-criteria analysis of electricity generation technologies in Lithuania. Renewable Energy, 85, 148-156. https://doi.org/10.1016/j.renene.2015.06.032

Trinkuniene, E., Podvezko, V., Zavadskas, E. K., Joksiene, I., Vinogradova, I., \& Trinkunas, V. (2017). Evaluation of quality assurance in contractor contracts by multi-attribute decision-making methods. Economic Research-Ekonomska Istrazivanja, 30(1), 1152-1180. https://doi.org/10.1080/1331677X.2017.1325616

Turskis, Z., \& Juodagalviene, B. (2016). A novel hybrid multi-criteria decision-making model to assess a stairs shape for dwelling houses. Journal of Civil Engineering and Management, 22(8), $1078-1087$. https://doi.org/10.3846/13923730.2016.1259179

Turskis, Z., Daniunas, A., Zavadskas, E. K., \& Medzvieckas, J. (2016). Multicriteria Evaluation of Building Foundation Alternatives. Computer- Aided Civil and Infrastructure Engineering, 31(9), 717-729. https://doi.org/10.1111/mice.12202

Turskis, Z., Zavadskas, E. K., Antucheviciene, J., \& Kosareva, N. (2015). A hybrid model based on fuzzy AHP and fuzzy WASPAS for construction site selection. International Journal of Computers Communications \& Control, 10(6), 113128. https://doi.org/10.15837/ijccc.2015.6.2078

Ulubeyli, S., \& Kazaz, A. (2016). Fuzzy multi-criteria decision making model for subcontractor selection in international construction projects. Technological and Economic Development of Economy, 22(2), $210-234$. https://doi.org/10.3846/20294913.2014.984363

Vasiljevic, M., Fazlollahtabar, H., Stevic, Z., \& Veskovic, S. (2018). A rough multicriteria approach for evaluation of supplier criteria in automotive industry. Decision Making: Applications in Management and Engineering, 1(1), 82-96. https://doi.org/10.31181/dmame180182v

Wang, Y. J., \& Lee, H. S. (2007). Generalizing TOPSIS for fuzzy multiple-criteria group decision making. Computers \& Mathematics with Applications, 53(11), 1762-1772. https://doi.org/10.1016/j.camwa.2006.08.037

Zadeh, L. A. (1965). Fuzzy sets. Information and Control, 8(3), 338-353. https://doi.org/10.1016/S0019-9958(65)90241-X

Zavadskas, E. K., Antucheviciene, J., Turskis, Z., \& Adeli, H. (2016). Hybrid multiple-criteria decision-making methods: A review of applications in engineering. Scientia Iranica. Transaction A: Civil Engineering, 23(1), 1-20. https://doi.org/10.24200/sci.2016.2093

Zavadskas, E. K., Turskis, Z., \& Bagocius, V. (2015). Multi-criteria selection of a deep-water port in the Eastern Baltic Sea. Applied Soft Computing, 26, 180-192. https://doi.org/10.1016/j.asoc.2014.09.019

Zavadskas, E. K., Turskis, Z., \& Kildiene, S. (2014). State of art surveys of overviews on MCDM/MADM methods. Technological and Economic Development of Economy, 20(1), 165-179. https://doi.org/10.3846/20294913.2014.892037

Zimmermann, H. J. (2010). Fuzzy set theory. Wiley Interdisciplinary Reviews: Computational Statistics, 2 (3), $317-332$. https://doi.org/10.1002/wics.82

The article has been reviewed.

Received in November, 2016; accepted in June, 2018. 